\title{
Affine Registration of Diffusion Tensor MR Images
}

\author{
Mika Pollari $^{1}$, Tuomas Neuvonen ${ }^{2,4}$, and Jyrki Lötjönen ${ }^{3}$ \\ ${ }^{1}$ Laboratory of Biomedical Engineering, Helsinki University of Technology, P.O.B. \\ 2200, FIN-02015 HUT, Finland \\ mika.pollari@tkk.fi \\ 2 Department of Clinical Neurophysiology, Helsinki University Central Hospital, \\ P.O.B. 340, FIN-00029 HUS, Finland \\ tuomas.neuvonen@hus.fi \\ ${ }^{3}$ VTT Information Technology, P.O.B. 1206, FIN-33101 Tampere, Finland \\ jyrki.lotjonen@vtt.fi \\ ${ }^{4}$ Neuroscience Unit, Dept. of Physiology, Inst. of Biomedicine, University of Helsinki
}

\begin{abstract}
We present a new algorithm for affine registration of diffusion tensor magnetic resonance (DT-MR) images. The method is based on a new formulation of a point-wise tensor similarity measure, which weights directional and magnitude information differently depending on the type of diffusion. The method is compared to a reference method, which uses normalized mutual information (NMI), calculated either from a fractional anisotropy (FA) map or a $T_{2}$-weighted MR image. The registration methods are applied to real and simulated DT-MR images. Visual assessment is done for real data and for simulated data, registration accuracy is defined. The results show that the proposed method outperforms the reference method.
\end{abstract}

\section{Introduction}

Diffusion tensor magnetic resonance imaging (DT-MRI) 1 is a relatively novel method, which increases the diagnostic value of magnetic resonance imaging (MRI). DT-MRI is based on the attenuation of MR signal due to the random motion of water molecules during a particular diffusion-weighted imaging sequence. DT-MRI assumes a Gaussian diffusion profile and models the diffusion of water molecules in tissue with diffusion tensors, which can be estimated from a set of diffusion-weighted MR images. In the brain, the diffusion process varies greatly between tissues. In the cerebro-spinal fluid (CSF) and in most parts of the gray matter (GM) diffusion is isotropic, i.e. without a preferred direction. The white matter (WM) consists of axonal fiber bundles, which lead on to anisotropic diffusion, where the principal diffusion direction is parallel to the local orientation of fibers. Same behavior is also seen in thalamus and in some other internal GM structures. The ability of DT-MRI to detect white matter abnormalities has made it a popular method in neuroimaging applications. In 2002 Kubicki et al. reported that DT-MRI had been used in 14 different clinical applications such 
as multiple sclerosis, Alzheimer's disease, and schizophrenia [2]. In this paper we study the affine registration of DT-MR data. The main motivation behind this study is the construction of DT-MR atlas, which requires inter-subject registration. DT-MR atlases provide a tool to compare variations in white matter between groups and between a group and a subject.

The simplest approach to DT-MR registration is to use the scalar data instead of the tensor data. The registration is based on either scalar indices or $T_{2}$-weighted MR images. Several indices have been proposed, see for instance 3 . The most frequently used indices are related to the size of diffusion (e.g. tensor trace), or the magnitude of anisotropy (e.g. fractional anisotropy). Even though scalar approach is simple, a lot of valuable information is discarded. Multi-channel registration methods present a second approach to DT-MR registration. Guimond et al. and Park et al. have introduced a multi-channel demons algorithm using the sum of squared differences calculated from the independent tensor components 4/5. Leemans et al. proposed an affine registration method using $k$-channel mutual information calculated from 60 diffusion weighted and one $T_{2}$-weighted MR image [6]. Rohde et al. introduced a nonlinear registration method using multivariate mutual information calculated from independent tensor components and $T_{2}$-weighted data 7 . Yet another type of approach is based on a direct similarity between tensors. Alexander et al. 88 have extended an elastic multi-resolution matching algorithm to DT data. Zhang et al. proposed a registration algorithm which is based on diffusion profiles 9. Ruiz-Alzola et al. [10] proposed a method, where registration is based on the identification of highly structured points and local matching of DT data.

In this work, we introduce a novel affine DT-MR registration method. The transformation is based on the global affine model. Our main contribution is the unique formulation of a point-wise tensor similarity measure. It measures the overlap between diffusion modes (i.e. linear, planar, or isotropic). This overlap is weighted with information, which is the most reliable in each mode.

The rest of the paper is organized as follows: In Sec. 2.1 the material is described. The general framework for the registration method is given in Sec. 2.2 Next, a detailed description of the point-wise similarity measure is given in Sec. 2.3. Evaluation protocol is described in Sec. 2.4. The results are presented in Sec. 3. Finally, the paper is finished with discussion and conclusion in Sec. 4

\section{Material and Methods}

\subsection{Material}

We acquired diffusion tensor images from 4 voluntary subjects, 2 males and 2 females, without any diagnosed neurological disorders (ages from 27 to 30 years). The data were acquired with a Siemens Sonata 1.5T MRI scanner (Siemens, Erlangen, Germany) at the Helsinki University Central Hospital using $128 \times 128$ matrix, $1.75 \times 1.75 \mathrm{~mm}^{2}$ in plane resolution, $4 \mathrm{~mm}$ slice thickness and $12 \mathrm{non}$ collinear diffusion weighting gradients with a $b$-value of $1000 \mathrm{~s} / \mathrm{mm}^{2}$, echo time (TE) of $119 \mathrm{~ms}$, repetition time (TR) of $6800 \mathrm{~ms}$, collecting 36 contiguous slices, 
repeating the measurement 6 times. Diffusion tensors were estimated for each voxel using a least-squares approach [11. All measurements were approved by the local ethical committee.

\section{$2.2 \quad$ Registration Method}

The goal in registration was to find an optimal transformation $T^{*}: x \rightarrow x^{\prime}$ which transformed the spatial coordinates of the source volume $A$ to the spatial coordinates of the reference volume $B$, so that the similarity function $I$ was maximized

$$
T^{*}=\max _{T} I(A(T(x)), B(x)) .
$$

The voxels from the source volume were transformed to the co-ordinate system of the destination volume using an affine transformation

$$
T(x)=\mathbf{M} x+\mathbf{t},
$$

where $\mathbf{M}$ was a $[3 \times 3]$ matrix and $\mathbf{t}$ was the translation vector.

During the transformation tensors were reorientated to ensure that their orientation remained consistent with the anatomical surrounding. In this study we used Preservation of principal direction (PPD) reorientation strategy, see details in 12 . In PPD reorientation two rotation matrices $\mathbf{R}_{1}$ and $\mathbf{R}_{2}$ were constructed and each transformed tensor $\mathbf{D}$ was reoriented using $\mathbf{D}^{\prime}=\left(\mathbf{R}_{2} \mathbf{R}_{1}\right) \mathbf{D}\left(\mathbf{R}_{2} \mathbf{R}_{1}\right)^{T}$. The rotation matrices were constructed from the known rotation axis and angle. First, the matrix $\mathbf{M}$ was applied to unit eigenvectors $\mathbf{v}_{1,2,3}$ and new unit vectors $\mathbf{n}_{i}=\mathbf{M} \mathbf{v}_{i} /\left|\mathbf{M} \mathbf{v}_{i}\right|$ were calculated. The first rotation matrix $\mathbf{R}_{1}\left(\mathbf{r}_{1}, \theta_{1}\right)$ mapped $\mathbf{v}_{1}$ onto $\mathbf{n}_{1}$. The rotation axis $\mathbf{r}_{1}$ and the rotation angle $\theta_{1}$ for $\mathbf{R}_{1}$ were

$$
\mathbf{r}_{1}=\mathbf{v}_{1} \times \mathbf{n}_{1}, \quad \theta_{1}=\arccos \left(\mathbf{v}_{1} \cdot \mathbf{n}_{1}\right) .
$$

In (31), ' $x$ ' and '.' denoted cross and dot product. The projection of $\mathbf{n}_{2}$ onto plane perpendicular of $\mathbf{R}_{1} \mathbf{v}_{1}$ was

$$
\mathbf{P}\left(\mathbf{n}_{2}\right)=\mathbf{n}_{2}-\left(\mathbf{n}_{2} \cdot \mathbf{n}_{1}\right) \cdot \mathbf{n}_{1} .
$$

The second rotation matrix $\mathbf{R}_{2}\left(\mathbf{r}_{2}, \theta_{2}\right)$ mapped $\mathbf{v}_{2}$ from the position after first rotation $\mathbf{R}_{1} \mathbf{v}_{2}$ onto plane spanned by $\mathbf{n}_{1}$ and $\mathbf{n}_{2}$. The rotation axis $\mathbf{r}_{2}$ and the rotation angle $\theta_{2}$ for $\mathbf{R}_{2}$ were

$$
\mathbf{r}_{2}=\mathbf{R}_{1} \mathbf{v}_{2} \times \mathbf{P}\left(\mathbf{n}_{2}\right) /\left|\mathbf{P}\left(\mathbf{n}_{2}\right)\right|, \quad \theta_{2}=\arccos \left(\mathbf{R}_{1} \mathbf{v}_{2} \cdot \mathbf{P}\left(\mathbf{n}_{2}\right) /\left|\mathbf{P}\left(\mathbf{n}_{2}\right)\right|\right) .
$$

In the location where the source voxel was mapped, a new value for the destination data was interpolated. For scalar data we used tri-linear interpolation and for tensors we used Log-Euclidean tri-linear interpolation [13.

$$
\hat{\mathbf{D}}=\exp \left(\sum_{i=1}^{8} w_{i} \log \left(\mathbf{D}_{i}\right)\right)
$$


where $w_{i}$ were the weights for tri-linear interpolation. Log-Euclidean interpolation was fast to compute and it produced positive definite tensors.

The similarity measure was calculated voxel-by-voxel between the transformed and reoriented source voxels and the interpolated destination voxels, see details in Sec. 2.3. The similarity measure was calculated only from the brain voxels. The brain tissue was extracted using the following simple routine: 1) Thresholding was done for $T_{2}$ data. Thresholding removed all air voxels leaving the actual brain voxels and some unwanted voxels (skin, fat, etc.) untouched. 2) The morphological opening was done in order to separate the unwanted voxels from the brain. 3) Connected components were labeled and the largest component (brain) was kept. 4) The morphological closing was performed to cancel out the effects of morphological opening. As an optimization, downhill simplex method from the hand-book of Numerical Recipes in C [14] was used.

\subsection{Similarity Measure}

Our approach was based on deriving a point-wise similarity measure. We looked overlap between diffusion modes (linear, planar, isotropic), and weighted this overlap with the information, which was the most reliable in that mode. For the diffusion modes, we used the coefficients defined by Westin et al. in [15]

$$
c_{l}=\frac{\lambda_{1}-\lambda_{2}}{\lambda_{1}}, \quad c_{p}=\frac{\lambda_{2}-\lambda_{3}}{\lambda_{1}}, \quad c_{s}=\frac{\lambda_{3}}{\lambda_{1}},
$$

where subscripts $l, p, s$ denoted linear, planar, and spherical (i.e. isotropic) diffusion, and $\lambda_{1,2,3}$ were the tensor eigenvalues.

With these notations the similarity between reference $A$ and source $B$ volume had the form

$$
I(A, B)=\sum_{a \in \Omega_{A}, b \in \Omega_{B}} c_{l}^{a} c_{l}^{b} S_{l}(a, b)+c_{p}^{a} c_{p}^{b} S_{p}(a, b)+\gamma * c_{s}^{a} c_{s}^{b} S_{s}(a, b),
$$

where $S_{l, p, s}$ were the mode-dependent similarity functions, $\Omega_{A, B}$ were the brain masks for the volumes, $\gamma$ was a weighting coefficient, and superscript $a$ denoted the transformed and reoriented source voxel, and superscript $b$ denoted the corresponding interpolated destination voxel. Isotropic diffusion (CSF, GM, WM) was more common in brain than the anisotropic diffusion (only in WM). As we wanted to accurately align also fibers in the WM, we had to weight less isotropic voxels, and thus the weighting $\gamma$ was introduced 1 . Next, we derived mode-dependent similarity functions $S_{l, p, s}$. In the case of isotropic diffusion, the tensor trace $t r=\frac{\lambda_{1}+\lambda_{2}+\lambda_{3}}{3}$, and gray-level $(g)$ from $T_{2}$ data were well defined, whereas the directional information was ambiguous. Thus, we proposed the following similarity measure for isotropic diffusion:

$$
S_{s}(a, b)=\frac{1}{2}\left(\left(1-\frac{\left|t r^{a}-t r^{b}\right|}{\max \left(t r^{a}, t r^{b}, 1\right)}\right)+\left(1-\frac{\left|g^{a}-g^{b}\right|}{\max \left(g^{a}, g^{b}, 1\right)}\right)\right) .
$$

\footnotetext{
${ }^{1}$ In all the experiments, we used value $\gamma=0.5$.
} 
In the case of linear and planar diffusion, the tensor trace and the gray-level information were useless as we already knew the tissue class (anisotropic diffusion took place in $\mathrm{WM} 2$ ). For linear diffusion, the first eigenvector $\mathbf{v}_{1}$ was well-defined whereas the other eigenvectors were ambiguous. Similarly for the planar diffusion, the third eigenvector $\mathbf{v}_{3}$ was well-defined but the other directions were ambiguous. Thus, we proposed the following directional similarities for linear and planar diffusion

$$
S_{l}(a, b)=\left|\mathbf{v}_{1}^{a} \cdot \mathbf{v}_{1}^{b}\right|, \quad S_{p}(a, b)=\left|\mathbf{v}_{3}^{a} \cdot \mathbf{v}_{3}^{b}\right|,
$$

(where '.' denoted the dot product). Equations (7)-(10) were combined and the similarity function was derived

$$
\begin{aligned}
I(A, B)= & \sum_{a \in \Omega_{A}, b \in \Omega_{B}} c_{l}^{a} c_{l}^{b}\left|\mathbf{v}_{1}^{a} \cdot \mathbf{v}_{1}^{b}\right|+c_{p}^{a} c_{p}^{b}\left|\mathbf{v}_{3}^{a} \cdot \mathbf{v}_{3}^{b}\right|+ \\
& +\gamma * c_{s}^{a} c_{s}^{b} \frac{1}{2}\left(\left(1-\frac{\left|t r^{a}-t r^{b}\right|}{\max \left(t r^{a}, t r^{b}, 1\right)}\right)+\left(1-\frac{\left|g^{a}-g^{b}\right|}{\max \left(g^{a}, g^{b}, 1\right)}\right)\right)
\end{aligned}
$$

For a reference to our similarity measure we used normalized mutual information [16]. NMI was calculated either from the $T_{2}$ data or from the FA maps

$$
F A=\frac{\sqrt{3\left(\left(\lambda_{1}-\bar{\lambda}\right)^{2}+\left(\lambda_{2}-\bar{\lambda}\right)^{2}+\left(\lambda_{3}-\bar{\lambda}\right)^{2}\right)}}{\sqrt{2\left(\lambda_{1}^{2}+\lambda_{2}^{2}+\lambda_{3}^{2}\right)}} \text {, where } \bar{\lambda}=\frac{1}{3} \sum_{i=1}^{3} \lambda_{i} .
$$

\subsection{Evaluation}

We used a two stage evaluation protocol: 1) For real data we did visual assessment. Each subject was registered to the other subjects (a total of 12 registrations). The registered source volume was compared with the reference volume using the chessboard visualization. In the chessboard visualization a new volume was constructed, where every second cube (we used cube size $16 \times 16 \times 16$ voxels) was taken from the reference volume and every second cube from the transformed source volume. Misregistrations were seen as a disconnectivity in the cube borders. We divided the results into successful and misregistrations using visual (subjective) assessment. Using these classifications we calculated a success rate (i.e. percentage of successful registrations) for each method.

2) For simulated data we generated random affine transformations $T^{\text {known }}$. The matrix $\mathbf{M}$ was generated as

$$
\mathbf{M}=\mathbf{P}\left(p_{x}, p_{y}, p_{z}\right) \mathbf{Q}\left(\varphi_{x}, \varphi_{y}, \varphi_{z}\right) \mathbf{R}_{x}(\theta) \mathbf{R}_{y}(\omega) \mathbf{R}_{z}(\phi),
$$

where $\mathbf{P}$ wad a scaling matrix of form $\mathbf{P}=\operatorname{diag}\left(p_{x}, p_{y}, p_{z}\right), \mathbf{R}_{x, y, z}$ were rotation matrices around $x, y$ and $z$ axis, and $\mathbf{Q}$ was a shearing matrix of form

$$
\mathbf{Q}\left(\varphi_{x}, \varphi_{y}, \varphi_{z}\right)=\left[\begin{array}{ccc}
1 & \tan \left(\varphi_{z}\right) & \tan \left(\varphi_{y}\right) \\
1 & \tan \left(\varphi_{x}\right) \\
0 & 0 & 1
\end{array}\right]
$$

\footnotetext{
${ }^{2}$ And in some small internal GM structures.
} 


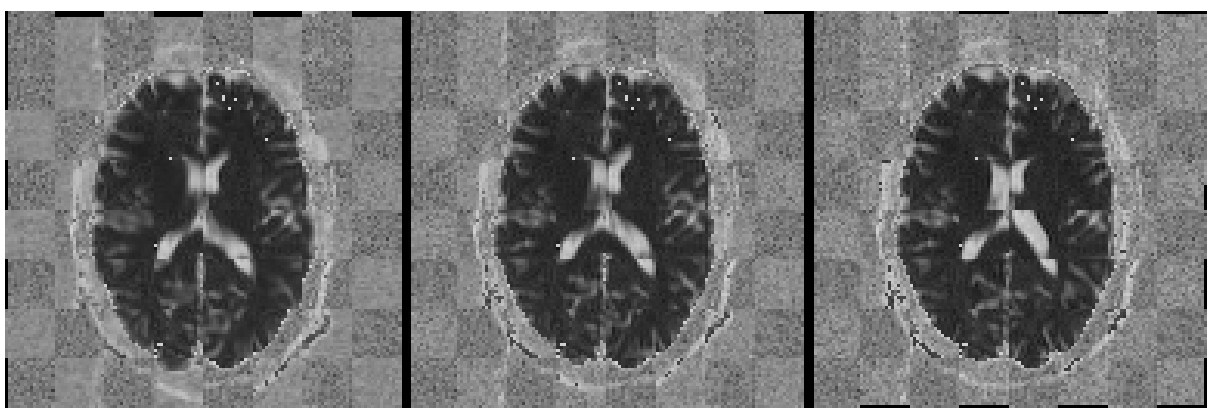

Fig. 1. The registration results for the proposed (left) and the reference method using MR images (middle) and FA maps (right) are shown using the chessboard visualization

The scaling parameters $p_{x, y, z}$ were randomly drawn from the uniform distribution $U(0.7,1.3)$, the shearing parameters $\varphi_{x, y, z}$ from the uniform distribution $U(-\pi / 8, \pi / 8)$, and the rotation angles $\theta, \omega, \phi$ from the uniform distribution $U(-\pi / 20, \pi / 20)$. Also translation parameters to each direction were drawn from the uniform distribution $U(-7,7)$ (measured in $\mathrm{mm}$ ). Each subject was transformed according to these known transformations. First, inverse transformation for $T^{\text {known }}$ was calculated. Using the inverse transformation we calculated the mapping from each voxel location of new volume (simulated data) to original data. A new value was interpolated in this location. We used tri-linear interpolation for scalar data and Log-Euclidean interpolation tensors. Interpolated voxels were transformed to new volume and during the transformation tensors were reoriented (PPD). As a result we obtained the simulated data, where we knew the ground truth transformations. The simulated data were registered to the original data $T^{r e g}$ and the registration error was calculated for all brain voxels (a total of $N$ voxels in $\Omega$ )

$$
\epsilon=\frac{1}{N} \sum_{x_{i} \in \Omega}\left\|\left(T^{r e g} \circ T^{k n o w n}\right) x_{i}-x_{i}\right\|_{2},
$$

\section{Results}

In Figure 1, the results were shown in one registration for the proposed (left) and the reference method using either $T_{2}$ images (middle) or FA maps (left). In the left and in the middle there were no notable errors. In the right, there was a clear misalignment. The results in the left and in the middle were

Table 1. The success rates for the proposed and the reference method using either MR images or FA maps. The total number of registrations was 12 .

\begin{tabular}{ll} 
Proposed NMI (MR) NMI (FA) \\
\hline $92 \% \quad 75 \% \quad 25 \%$ \\
\hline
\end{tabular}


classified as a successful registration, but the case in the right was classified as a misregistration. The success rates for real data were given in Table 1 .

Ten random affine transformations were applied to each subject and 40 simulated data sets were created. Using these data sets we calculated the registration accuracy for each method. The results were given in Table 2 ,

Table 2. The mean and the standard deviation of the registration accuracy (in $\mathrm{mm}$ ) for the simulated data

\begin{tabular}{ll} 
Proposed NMI (MR) NMI (FA) \\
\hline \begin{tabular}{lll}
$0.1 \pm 0.1$ & $8.9 \pm 8.9$ & $16.9 \pm 4.1$ \\
\hline
\end{tabular}
\end{tabular}

\section{Discussion and Conclusions}

The proposed and the reference method were first applied to real DT-MR data. The proposed method gave a good alignment in 11 registrations out of 12 (a success rate of $92 \%$ ). For the NMI-based reference method the success rate was considerably lower. With $T_{2}$ images the success rate was $75 \%$ and with FA maps only $25 \%$. Next, the methods were applied to the simulated data. The proposed method had the best robustness and accuracy (residual error $0.1 \pm 0.1 \mathrm{~mm}$.). When NMI-based reference method was used with $T_{2}$ data we noticed that there were large variations in the registration results (standard deviation 8.9 $\mathrm{mm}$ ). Thus the overall accuracy was poor (mean accuracy $8.9 \mathrm{~mm}$.). However, in 20 registrations out of 40 the average misregistration was smaller than the largest voxel dimension $(4.0 \mathrm{~mm})$. If only these registrations were considered, the method had a reasonable registration accuracy $(0.4 \pm 0.8 \mathrm{~mm}$.). The results for FA map based NMI registration are poor (residual error $16.9 \pm 4.1$ ). Even the smallest individual registration error was as high as $12.5 \mathrm{~mm}$.

These tests demonstrated that the proposed method was more robust than the reference NMI-base reference method. Furthermore, the results showed that the FA map based NMI registration could not produce accurate registrations. It should be noted that we could not conclude from the experiments what was the (true) inter-subject registration accuracy. The problem was in the evaluation protocol. In real data registration, inter-subject variations were present. However, using visual assessment we could only define the success rates but not the registration accuracies. With the simulated data the registration accuracies were calculated. However, the simulated data didn't represent inter-subject variations. Thus, the simulated data experiment gave information about the robustness, but did not give any information about the (true) inter-subject registration accuracies.

In summary, we proposed a new method for DT-MR registration. Our main contribution was a new formulation of a point-wise tensor similarity measure. Although not demonstrated in this study, the developed similarity measure is not limited to affine transformations. In the future, we plan to extend the method to non-rigid and nonlinear transformations, make quantitative evaluation of the method, and compare the method to other DT-MR registration methods. 


\section{References}

1. Basser, P., Mattiello, J., LeBihan, D.: MR diffusion tensor spectroscopy and imaging. Biophys. J. 66 (1994) 259-267

2. Kubicki, M., Westin, C.F., Maier, S., H. Mamata, M.F., Ersner-Hershfield, H., Kikinis, R., Jolesz, F., McCarley, R., Shenton, M.E.: Diffusion Tensor Imaging and Its Application to Neuropsychiatric Disorders. Harvard Rev Psychiatry 10 (2002) 324-336

3. Basser, P., Pierpaoli, C.: Microstructural and Physiological Features of Tissue Elucidated by Quantitative-Diffusion-Tensor MRI. Journal of Magnetic Resonance, Series B 111 (1996) 209-219

4. Guimond, A., Guttmann, C.R.G., Warfield, S.K., Westin, C.F.: Deformable registration of DT-MRI data based on transformation invariant tensor characteristics. In: Proceedings of the IEEE International Symposium on Biomedical Imaging (ISBI'02), Washington (DC), USA (2002)

5. Park, H., et al.: Spatial normalization of diffusion tensor MRI using multiple channels. NeuroImage 20 (2003) 1995-2009

6. Leemans, A., Sijbers, J., Backer, S., Vandervliet, E., Parizel, P.: Affine Coregistration of Diffusion Tensor Magnetic Resonance Images Using Mutual Information. Lecture Notes in Computer Science 3708 (2005) 523-530

7. Rohde, G.K., Pajevic, S., Pierpaoli, C.: Multi-channel registration of diffusion tensor images using directional information. In: ISBI, IEEE (2004) 712-715

8. Alexander, D.C., Gee, J.C.: Elastic Matching of Diffusion Tensor Images. Computer Vision and Image Understanding 77 (2000) 233-250

9. Zhang, H., Yushkevich, P.A., Gee, J.C.: Deformable Registration of Diffusion Tensor MR Images with Explicit Orientation Optimization. In Duncan, J.S., Gerig, G., eds.: MICCAI. Volume 3749 of Lecture Notes in Computer Science., Springer (2005) $172-179$

10. Ruiz-Alzola, J., Westin, C.F., Warfield, S., Alberola, C., Maier, S., Kikinis, R.: Nonrigid registration of 3D tensor data. Medical Image Analysis 6 (2002) 143-161

11. Papadakis, N.G., Xing, D., Huang, C.L.H., Hall, L.D., Carpenter, T.A.: A Comparative Study of Acquisition Schemes for Diffusion Tensor Imaging Using MRI. Journal of Magnetic Resonance 137 (1999) 67-82

12. Alexander, D., Pierpaoli, C., Gee, P.B.J.: Spatial transformations of diffusion tensor images. IEEE Transactions on Medical Imaging 20 (2001) 1131-1139

13. Arsigny, V., Fillard, P., Pennec, X., Ayache, N.: Fast and simple computations on tensors with Log-Euclidian metrics. Research Raport 5584, INRIA (2005)

14. Press, W., Teukolsky, S., Vetterling, W., Flannery, B.: Numerical recipes in C: the art of scientific computing. Second edn. Cambridge University Press (1992)

15. Westin, C.F., Maier, S., Mamata, H., Nabavi, A., Jolesz, F., Kikinis, R.: Processing and visualization of diffusion tensor MRI. Medical Image Analysis 6 (2002) 93-108

16. Studholme, C., Hill, D.L., Hawkes, D.J.: An overlap invariant entropy mesure of 3D medical image aligment. Pattern Recognition 32 (1999) 71-86 